\title{
LA-UR-17-27100
}

Approved for public release; distribution is unlimited.

Title: $\quad$ DRiFT Stilbene Simulations for the NEUANCE Detector Array

Author(s): $\quad$ Pinilla, Maria Isabel

Andrews, Madison Theresa

Rising, Michael Evan

Intended for: Keepin Summer Program Presentation

Issued: 
Disclaimer:

Los Alamos National Laboratory, an affirmative action/equal opportunity employer, is operated by the Los Alamos National Security, LLC for the National Nuclear Security Administration of the U.S. Department of Energy under contract DE-AC52-06NA25396. By approving this article, the publisher recognizes that the U.S. Government retains nonexclusive, royalty-free license to publish or reproduce the published form of this contribution, or to allow others to do so, for U.S. Government purposes. Los Alamos National Laboratory requests that the publisher identify this article as work performed under the auspices of the U.S. Department of Energy. Los Alamos National Laboratory strongly supports academic freedom and a researcher's right to publish; as an institution, however, the Laboratory does not endorse the viewpoint of a publication or guarantee its technical correctness. 


\section{DRiFT Stilbene Simulations for the NEUANCE Detector Array}

Maria Isabel Pinilla ${ }^{1,2}$, Madison Andrews ${ }^{1}$, Mike Rising $^{1}$

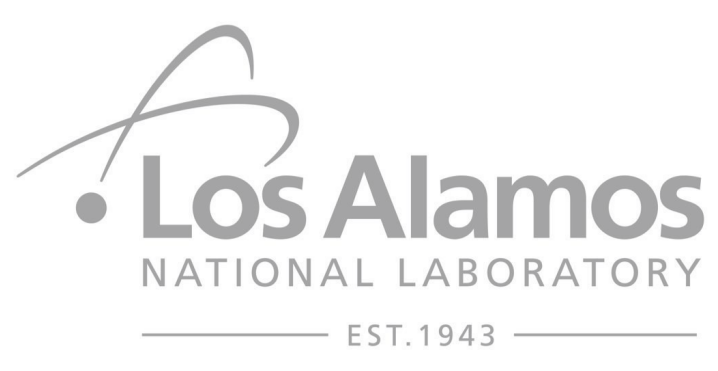

${ }^{1}$ Los Alamos National Laboratory XCP: Computational Physics Division XCP-3: Monte Carlo Methods, Codes, and Applications

${ }^{2}$ Kansas State University 


\section{Maria Isabel Pinilla (XCP-3)}

- Educational Background
o B.S. Mechanical Engineering, KSU, 2014
o M.S. Student

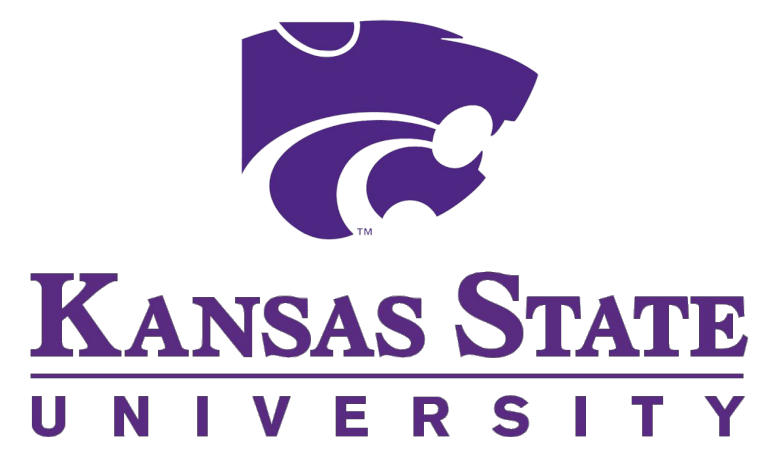

- XCP: Computational Physics Division

o XCP-3: Monte Carlo Methods, Codes, and Applications

○ Mentor: Madison Andrews
○ Co-mentor: Mike Rising
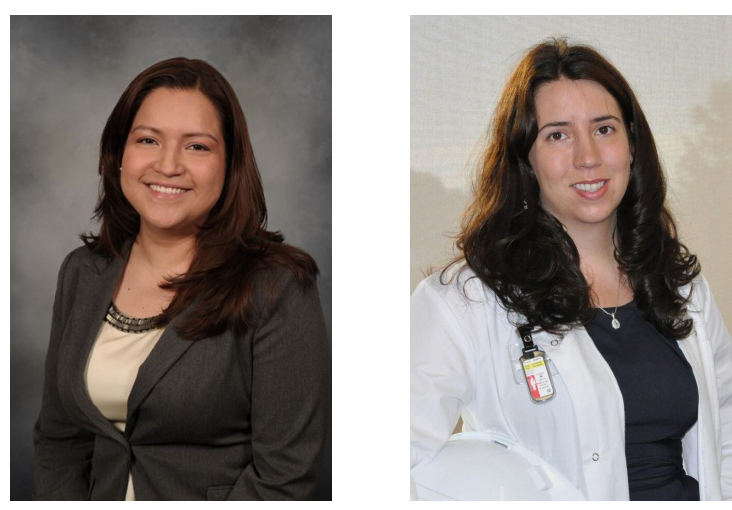

- Research

- Summer: Adding stilbene simulation capabilities to DRiFT

○ M.S.: Radiological source replacement for oil well logging applications 


\section{Research Overview and Motivation}

- Improve ability to simulate detector response functions through the use of MCNP associated tools

- MCNPTools

- Detector Response Function Toolkit (DRiFT)

- Currently under development

- Uses MCNPTools to read PTRAC files

- Generates detector response functions for scintillators

- Previously validated with EJ-301 measurements

- Stilbene research is gaining momentum in nonproliferation applications

- New crystal growth technique, larger crystals

- Higher light output and better PSD performance than EJ-309

- NEUANCE Detector Array at LANSCE

- Composed of 21 stilbene crystals

- Measurements at NEUANCE can provide validation for correlated fission and detector cross-talk models 


\section{Research Approach}

\section{- MCNP}

○ Light output function (DE/DF tally treatment card) - neutrons

- W. Hansen, NIM in Phys. Res., 2002

- M.M. Bourne, University of Michigan dissertation, 2016

- Gaussian Energy Broadening (GEB tally treatment card) - neutrons \& gammas

- Iterative MC simulation with Compton kinematics-based GEB approach, C. Kim, NIM, 2015

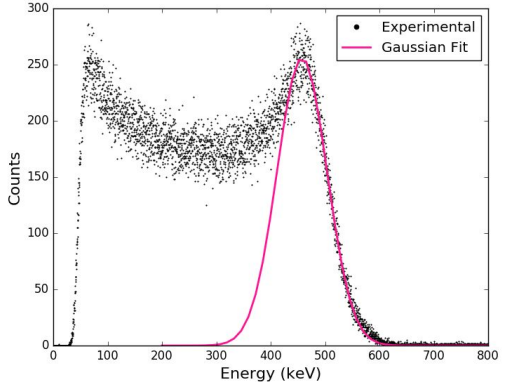

Fig 1: Gaussian fit to experimental spectrum

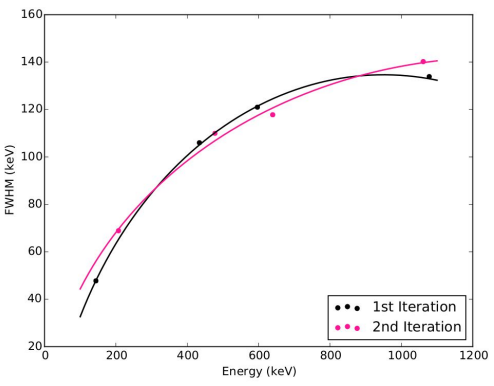

Fig 2: Iterative GEB fit

- DRiFT stilbene processing capabilities

- Light output function

- Light emission spectrum (a)

- Neutron and gamma pulse shapes (b)

- PMT quantum efficiency (c)

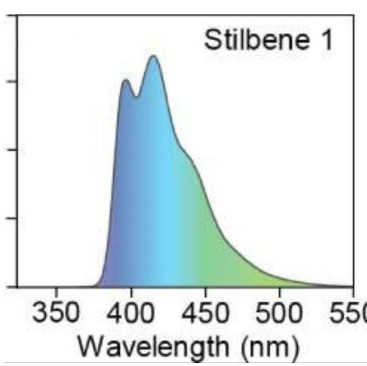

Fig 3: (a)
- GEB constants:
○ $a=-0.0572$
$\circ b=0.2497$
$\circ c=-0.4321$

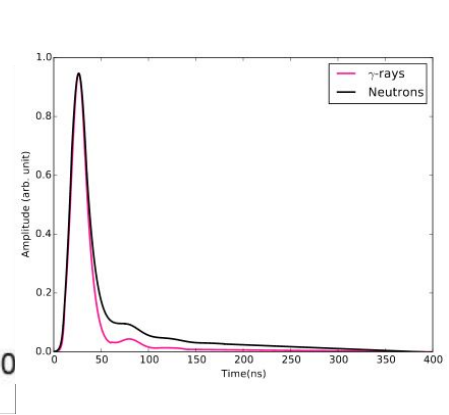

Fig 4: (b)

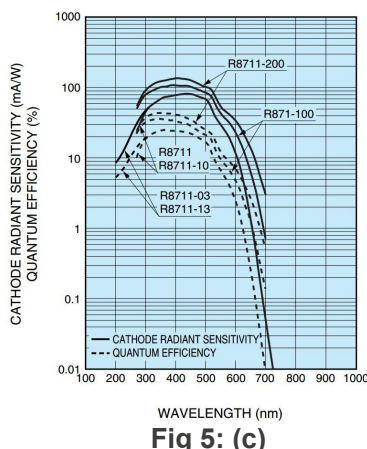




\section{Summary of Results}

\section{- Gamma-rays}

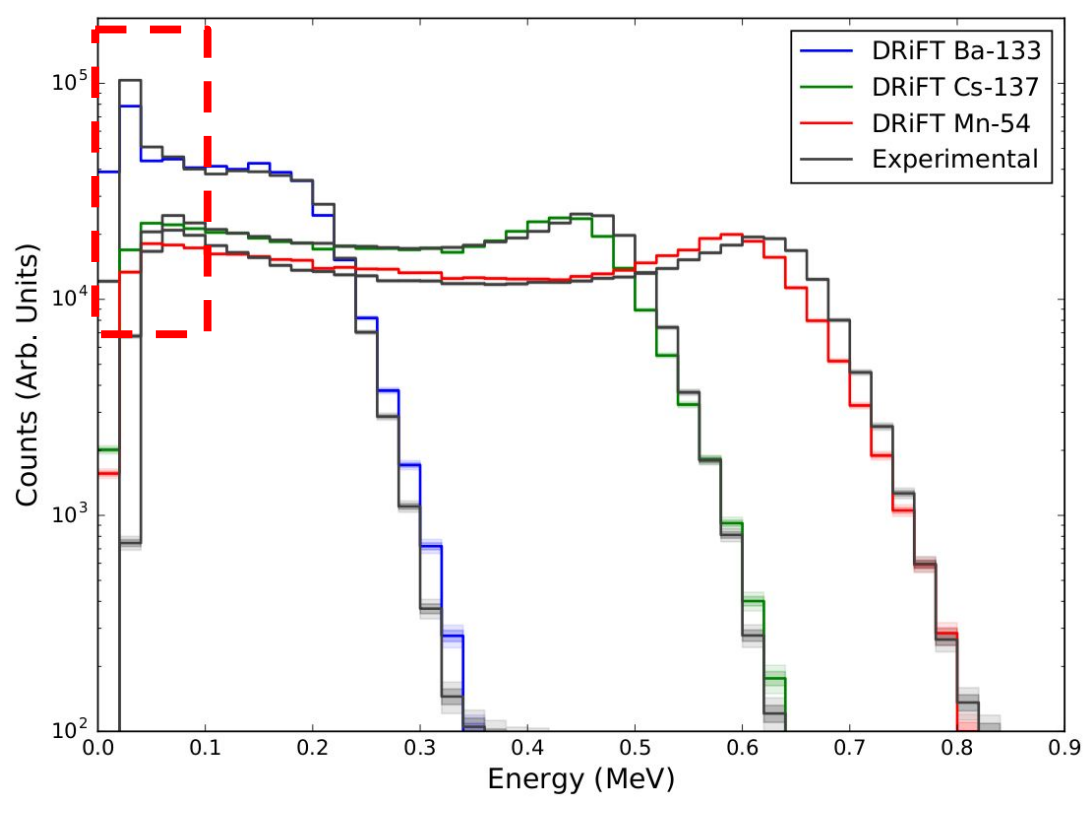

Fig 6: DRiFT Gamma-ray Results
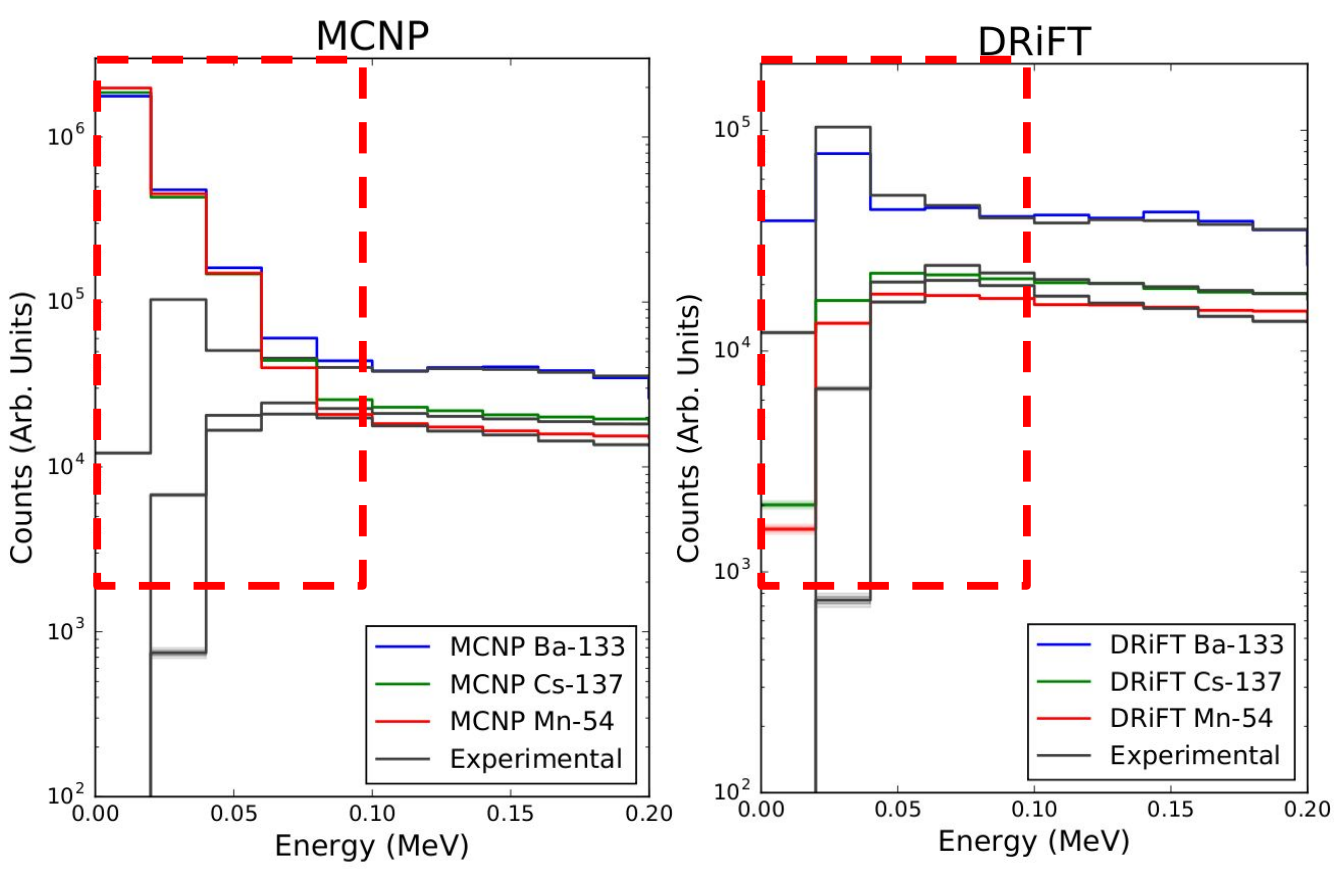

Fig 7: MCNP v. DRiFT Low-Energy Comparison 


\section{Summary of Results}

- Neutrons

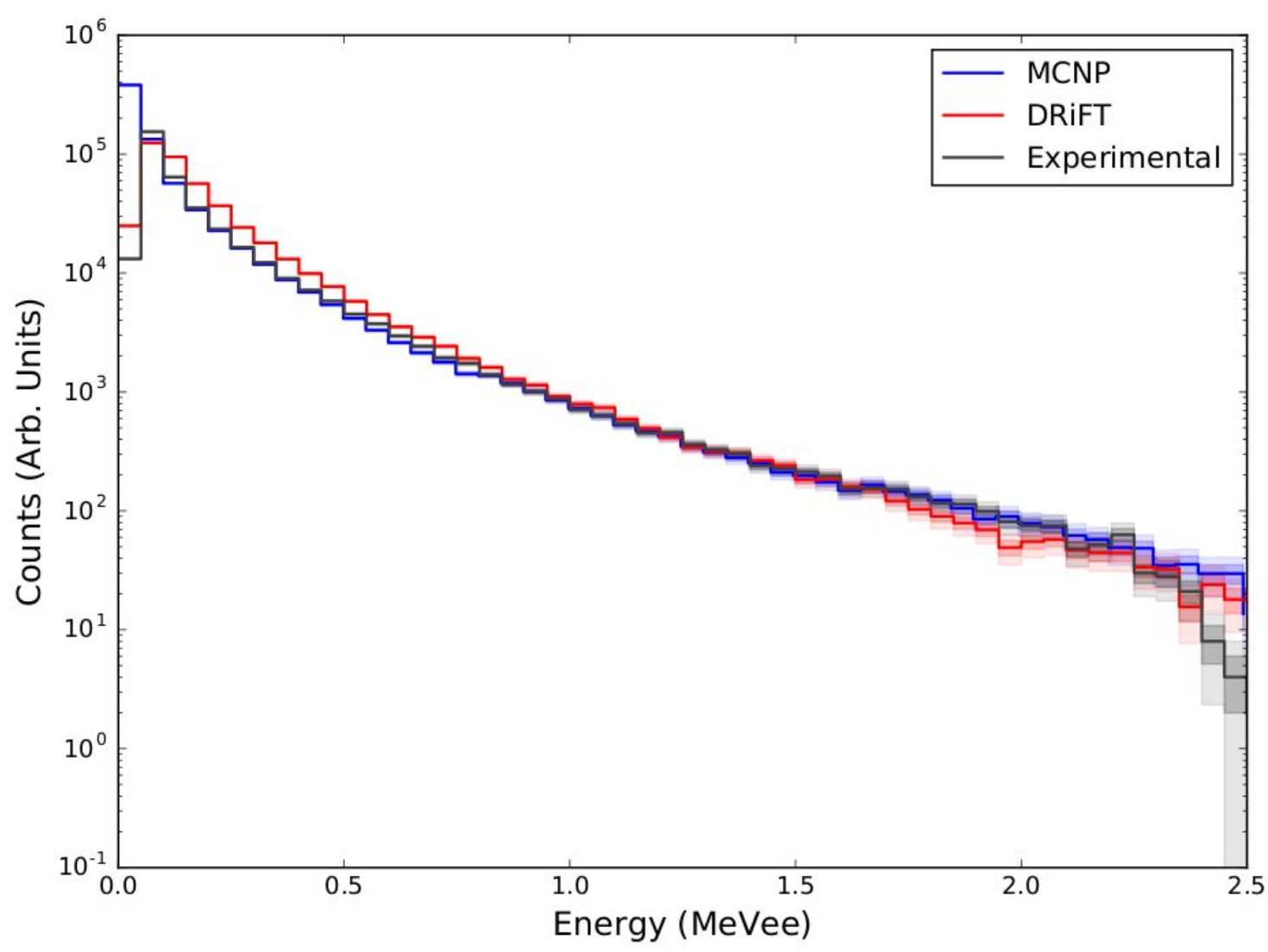

Fig 6: MCNP and DRiFT Cf-252 Simulation Results 


\section{Summer Fun}

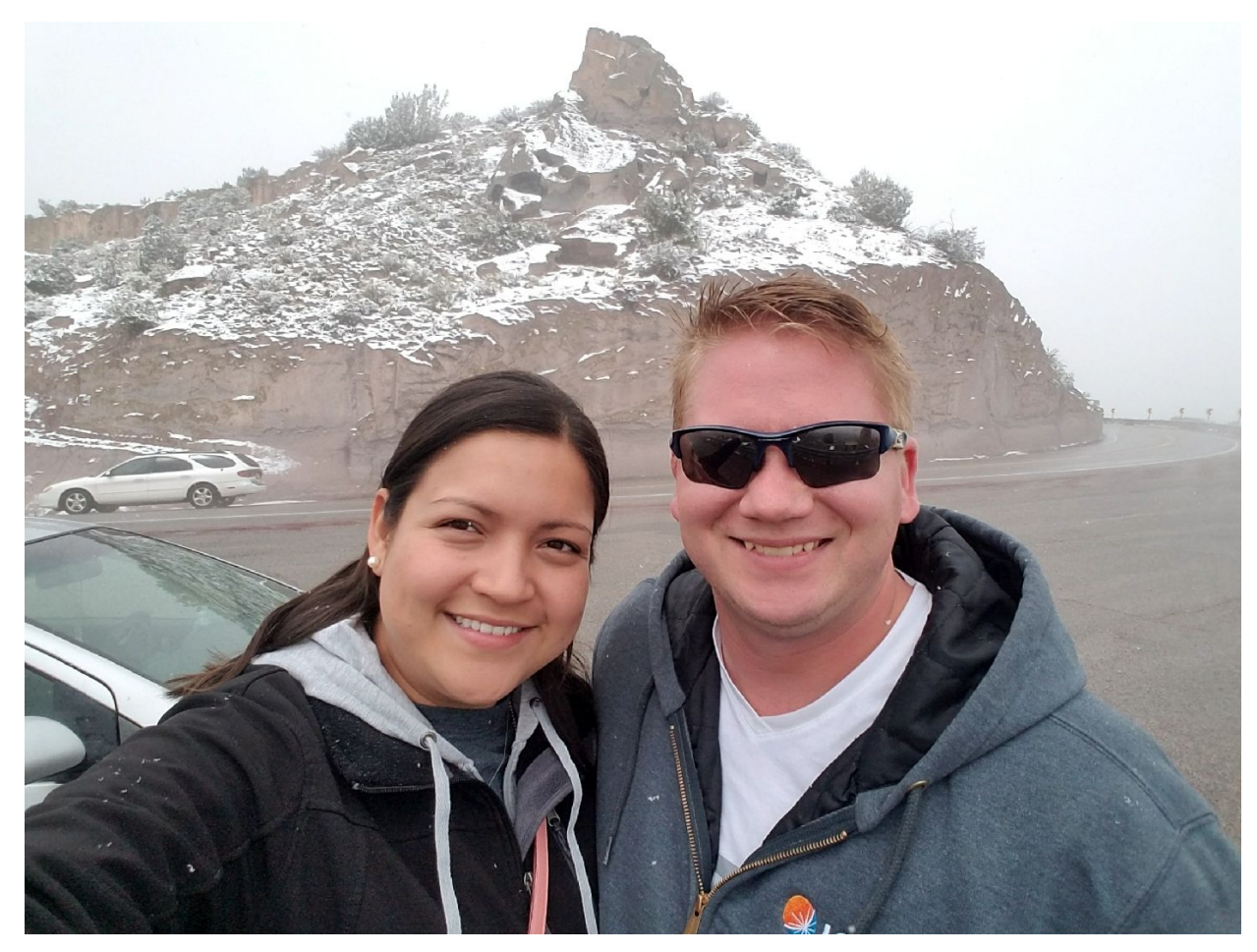

Snowing in May in Los Alamos

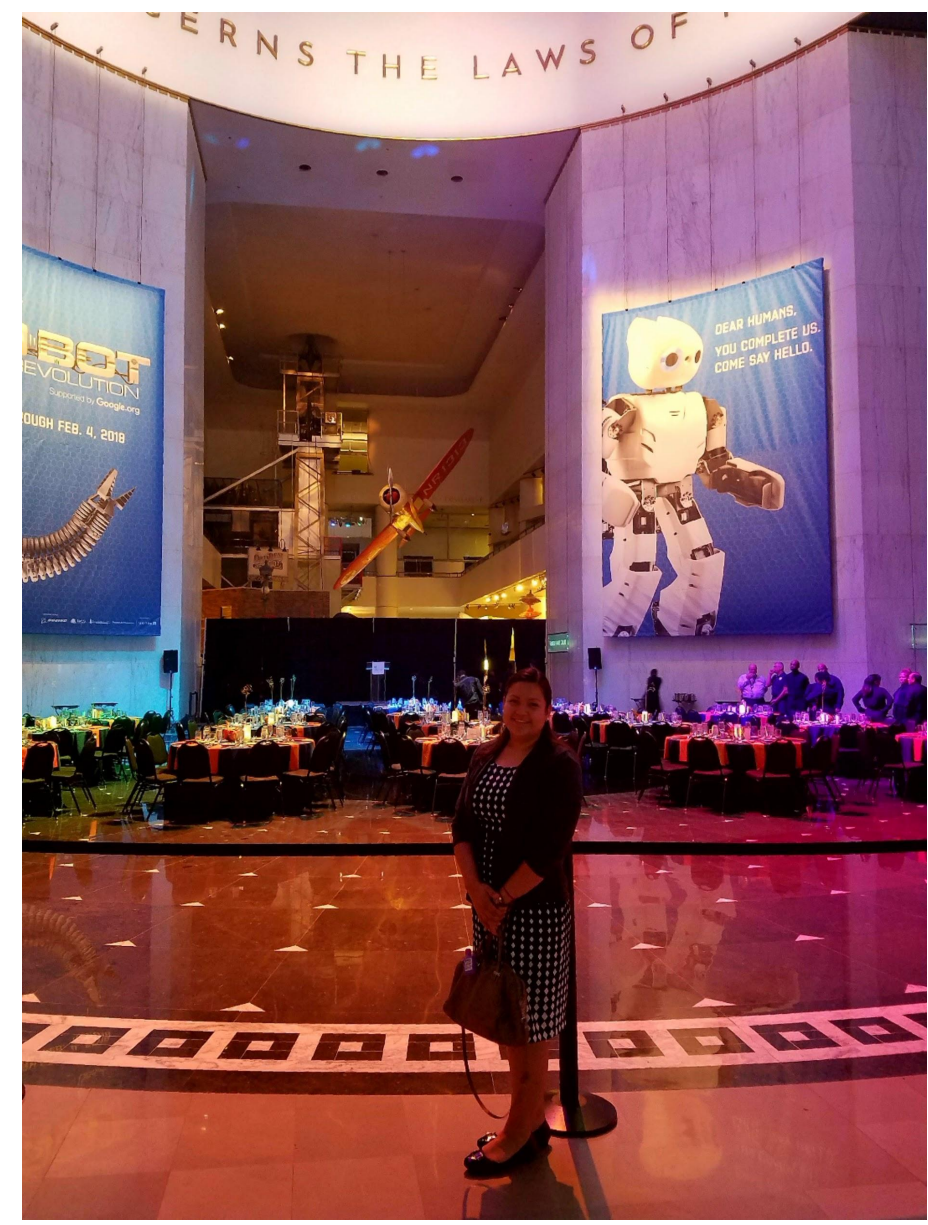

IRRMA X, Chicago, IL 